\title{
IRAS OBSERVATIONS OF SYMBIOTIC STARS
}

\author{
M. Parthasarathy H.C. Bhatt \\ Indian Institute of Astrophysics \\ Bangalore - 560034, India
}

\begin{abstract}
Of the 129 symbiotic stars in Allen's (1984) catalogue, 42 were found to be IRAS sources. Of these 42 IRAS sources, 22 are D-type (symbiotic Miras), 5 are $D^{\prime}$-type (yellow symbiotics) and 15 are S-type. The separation of $S, D$ and $D^{\prime}$ types into three distinct groups is clearer in the $\log [\mathrm{f} \lambda(25 \mu \mathrm{m}) / \mathrm{f} \lambda(12 \mu \mathrm{m})]$ versus $(\mathrm{H}-\mathrm{K})$ diagram. The IRAS fluxes of $S$-type symbiotics are consistent with that observed from normal M giants. This result suggests that mass-loss rate from most of the S-type symbiotics is similar to that from normal $M$ giants. The IRAS data of D-type symblotics shows evidence for the presence of dust envelopes. The masses of the dust envelopes $\left(10^{-6}\right.$ to $\left.10^{-7} \mathrm{Mo}\right)$ around Miras in D-type symbiotics are similar to that observed in field Mira variables. These results suggest that mass-loss rates in symbiotic Miras are similar to those from field Mira variables and also that the mass loss from symbiotic Miras is pulsationally driven similar to that found in field Mira variables by Whitelock. Pottasch and Feast (1987). Analysis of IRAS data of yellow symbiotics M1-2, AS201, Cn1-1, Wray 157 , and HD149427 suggests that they are young planetary nebulae containing a binary nucleus. M1-2. AS201 and Cn1-1 show evidence for the presence of evolved hot companions. The evolutionary stage of the late type (F-G) companions is not clear.
\end{abstract}

\section{INTRODUCTION}

The spectra of symbiotic stars show features commonly observed in late-type giants and high-excitation planetary nebulae. The late-type star in a symbiotic binary is typically a M-type giant or an AGB star or a Mira. The hot component of a symbiotic system is a hot white dwarf or probably the central star of a planetary nebula or an accretion disk surrounding a low mass main sequence star (Kenyon and Webbink, 1984). From the near-inf rared observations symbiotic stars have been classified into three types (Webster and Allen, 1975; Allen, 1982). The S-type (stellar) symbiotics have stellar IR continua (Tcolour $=3000 \mathrm{~K}$ ) corresponding to the associated $M$ giants. The D-type (dusty: Tcolour = $800 \mathrm{~K})$ symbiotics show the presence of circumstellar dust, and the cool component is a Mira variable. In the $D^{\prime}$-type systems (yellow symbiotics) the dust is cooler $(T<500 \mathrm{~K})$, and the cool component is of spectral type F-G.

Broad-band JHKL photometry has been obtained for nearly all symbiotics (Allen 1982; Kenyon and Gallagher 1983). However, 10 um and $20 \mu \mathrm{m}$ observations are available for only some bright symbiotics. The IRAS point source catalogue (Beichman et al. 1985) now provides 
Table 1: IRAS OBSERVATIONS OF SYMBIOTIC STARS

\begin{tabular}{|c|c|c|c|c|c|}
\hline & \multirow[b]{2}{*}{ IR type } & \multicolumn{4}{|c|}{ Observed Fluxes (Jansky) } \\
\hline & & $12 \mu \mathrm{m}$ & $25 \mu \mathrm{m}$ & $60 \mu \mathrm{m}$ & $100 \mu \mathrm{m}$ \\
\hline EG and & $S$ & 3.22 & 0.89 & $<1.05$ & \\
\hline $\mathrm{M} 1-2$ & $D^{\prime}$ & 1.79 & 2.59 & 1.53 & \\
\hline UV Aur & S & 52.6 & 15.1 & 2.51 & \\
\hline Wray 157 & $D^{\prime}$ & 1.15 & 1.33 & 0.58 & \\
\hline RX Pup & $\mathrm{D}$ & 204 & 101 & 12.1 & 6.9 \\
\hline AS 201 & $D^{\prime}$ & 1.22 & 2.84 & 2.14 & 1.08 \\
\hline $\mathrm{He} 2-38$ & $\mathrm{D}$ & 8.32 & 3.4 & $<3.72$ & \\
\hline SY Mus & $S$ & 0.84 & 0.35 & & \\
\hline BI Cru & D & 18.9 & 13.3 & $<11.6$ & \\
\hline SS 38 & D & 6.73 & 2.89 & 1.61 & \\
\hline RW Hya & $S$ & 0.88 & $<0.45$ & $<0.4$ & \\
\hline He2-104 & D & 9.35 & 8.62 & 5.98 & \\
\hline He2-106 & D & 33.9 & 21.0 & 4.61 & 29.3 \\
\hline $\mathrm{He} 2-127$ & $\mathrm{D}$ & 0.55 & 0.33 & $<0.68$ & \\
\hline Cn1-1 & $D^{\prime}$ & 23.1 & 35.6 & 15.2 & 9.23 \\
\hline $\mathrm{T} \mathrm{CrB}$ & $S$ & 0.61 & 0.24 & & \\
\hline AG Dra & $S$ & 0.3 & $<0.75$ & $<0.4$ & \\
\hline He2-147 & D & 4.07 & 2.39 & $<0.4$ & \\
\hline He2-171 & D & 7.36 & 3.54 & 0.61 & \\
\hline HD 149427 & $D^{\prime}$ & 2.65 & 8.16 & 1.45 & \\
\hline $\mathrm{HE} 2-176$ & $\mathrm{D}$ & 2.97 & 2.07 & & \\
\hline Hen 1242 & $S$ & 0.36 & $<0.25$ & & \\
\hline AS 210 & $\mathrm{D}$ ? & 3.05 & 0.9 & $<0.4$ & \\
\hline $\mathrm{H} 2-5$ & $D ?$ & 1.19 & 0.61 & & \\
\hline $\mathrm{H} 1-36$ & $D$ & $<18.2$ & 20.8 & 4.35 & \\
\hline AS 245 & $\mathrm{D} ?$ & 1.53 & 0.65 & & \\
\hline Apl-8 & S? & 0.85 & 0.53 & 3.14 & 34.4 \\
\hline SS 122 & $\mathrm{D}$ ? & 1.45 & 0.84 & & \\
\hline $\mathrm{H} 2-38$ & $D$ & 3.35 & 1.69 & & \\
\hline AS 296 & $S$ & 0.88 & 0.35 & $<0.4$ & \\
\hline $\mathrm{He} 2-390$ & D & 5.08 & 3.94 & 1.76 & 6.61 \\
\hline V3804 Sgr & $\mathrm{S}$ & 0.51 & $<0.51$ & $<0.44$ & \\
\hline V443 Her & S & 0.39 & $<0.25$ & $<0.4$ & \\
\hline $\mathrm{CH}$ Cyg & $S$ & 448 & 140 & 13.7 & 3.76 \\
\hline Hen 1761 & $S$ & 0.42 & $<0.27$ & $<0.4$ & \\
\hline HM Sge & $\mathrm{D}$ & 119 & 63.1 & 7.08 & \\
\hline CI Cyg & $S$ & 0.76 & 0.25 & $<0.45$ & \\
\hline V1016 Cyg & $\mathrm{D}$ & 45.1 & 26.9 & 3.03 & \\
\hline RR Tel & $\mathrm{D}$ & 21.1 & 12.9 & 1.98 & \\
\hline AG Peg & $S$ & 1.49 & 0.47 & $<0.42$ & \\
\hline 2 And & $S$ & 0.58 & 0.21 & $<0.37$ & \\
\hline R Aqr & $\mathrm{D}$ & 1262 & 401 & 50.5 & 14.8 \\
\hline
\end{tabular}


far-infrared fluxes from $12 \mu \mathrm{m}$ to $100 \mu \mathrm{m}$ for several symbiotic stars. The

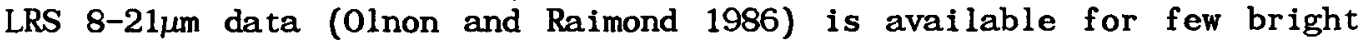
symbiotic stars. In this paper we report an analysis of the IRAS observations of symbiotic stars.

\section{IRAS OBSERVATIONS}

Allen's (1984) catalogue lists 129 symbiotic stars and 15 possible symbiotics. In Table 1 we list the IRAS observations of the symbiotics stars detected in the IRAS point source catlogue. The observed IRAS positions are in good agreement with their optical positions. The IRAS fluxes given in Table 1 have been colour corrected as described in the IRAS explanatory supplement. Only 8 symbiotic stars have LRS $8-21 \mu \mathrm{m}$ spectra (Figure 1).

\section{ANALYSIS}

Of the 129 symbiotic stars in the Allen's (1984) catalogue, 42 were found to be IRAS sources (Table 1): 22 are D-type, 5 are D'-type and 15 are S-type. Except $D^{\prime}$-type symbiotics, the D-type and S-type symbiotics show decreasing IR flux with increasing wavelength. Most of the S-types do not show significant flux at 60 $\mathrm{mm}$. Only $3 \mathrm{D}$-type and $5 \mathrm{D}$-type show flux at 100 $\mathrm{mm}$. He 2-106, Ap1-8, He2-390 show flux at 100 $\mathrm{mm}$. These three are close to the galactic plane, and the increased emission at $100 \mu \mathrm{m}$ may be due to IR cirrus. The flux ratio $f \lambda(12 \mu \mathrm{m}) / \mathrm{f} \lambda(25 \mu \mathrm{m})$ of $S$ and D-types range from 1 to 3 . This flux ratio from the data of $D^{\prime}$-type symbiotics is in the range of 0.3 to 0.9 . In figure 2 we show the flux ratios $\log [\mathrm{f} \lambda(12 \mu \mathrm{m}) / \mathrm{f} \lambda(2.2 \mu \mathrm{m})]$ versus $\log [\mathrm{f} \lambda(25 \mu \mathrm{m}) / \mathrm{f} \lambda(12 \mu \mathrm{m})]$. In Figure 2 the S-type symbiotics are in the same region as that defined by $M$ giants, and $\mathrm{D}$-types are in the same region as that defined by Mira variables. The D'-types appear to be well separated from $S$ and D-types. H2-5, SS122, Ap1-8 and AS245 are in the border region of D-type symbiotic stars (Figure 2).

Figure 3 shows the $(H-K)$ colour versus $\log [f \lambda(25 \mu \mathrm{m}) / f \lambda(12 \mu \mathrm{m})]$; there S,D, $D^{\prime}$-types are better separated than in Figure 2 . Figure 3 clearly shows the large spread in the $(\mathrm{H}-\mathrm{K})$ colours and relatively less spread in the $f \operatorname{lux}$ ratio $\log [\mathrm{f} \lambda(25 \mu \mathrm{m}) / \mathrm{f} \lambda(12 \mu \mathrm{m})]$ of $\mathrm{D}$-type symbiotics. From Figure 3 we clearly distinguish the five $D^{\prime}$-types as a distinct group. The $(\mathrm{H}-\mathrm{K})$ colour and the flux ratio $\log [\mathrm{f} \lambda(25 \mu \mathrm{m}) / \mathrm{f} \lambda(12 \mu \mathrm{m})]$ of SS122 suggests that it is a D-type symbiotic. SS122 appears to be similar to $\mathrm{He} 2-176$ and $\mathrm{He} 2-147$. He 2-147 was listed as a S-type symbiotic by Allen (1984). However, the position of He2-147 in Figures 2 and 3 suggests that it is most likely a symbiotic Mira. Ap1-8 and H2-5 are S-types; however, they appear to show excess flux at $12 \mu \mathrm{m}$ and $25 \mu \mathrm{m}$ compared to other S-types. AS245 also appears to show $12 \mu \mathrm{m}$ excess. It is listed as S-type, but its position in Figures 2 and 3 indicates that it may be a symbiotic Mira. The flux ratios of AS210 are similar to those of UV Aur and R Aqr. It is likely that AS210 may contain a carbon star or a symbiotic Mira. The IR types (S,D,D') based on Figures 2 and 3 and the far-infrared energy distributions of $S$ type symbiotics are consistent with that expected from normal field M giants.

From IRAS data of S-type symbiotic stars we can conclude that they do not have dust envelopes similar to that observed in symbiotic Miras. These results suggest that the mass-loss rate in $\mathrm{S}$-type symbiotics is 

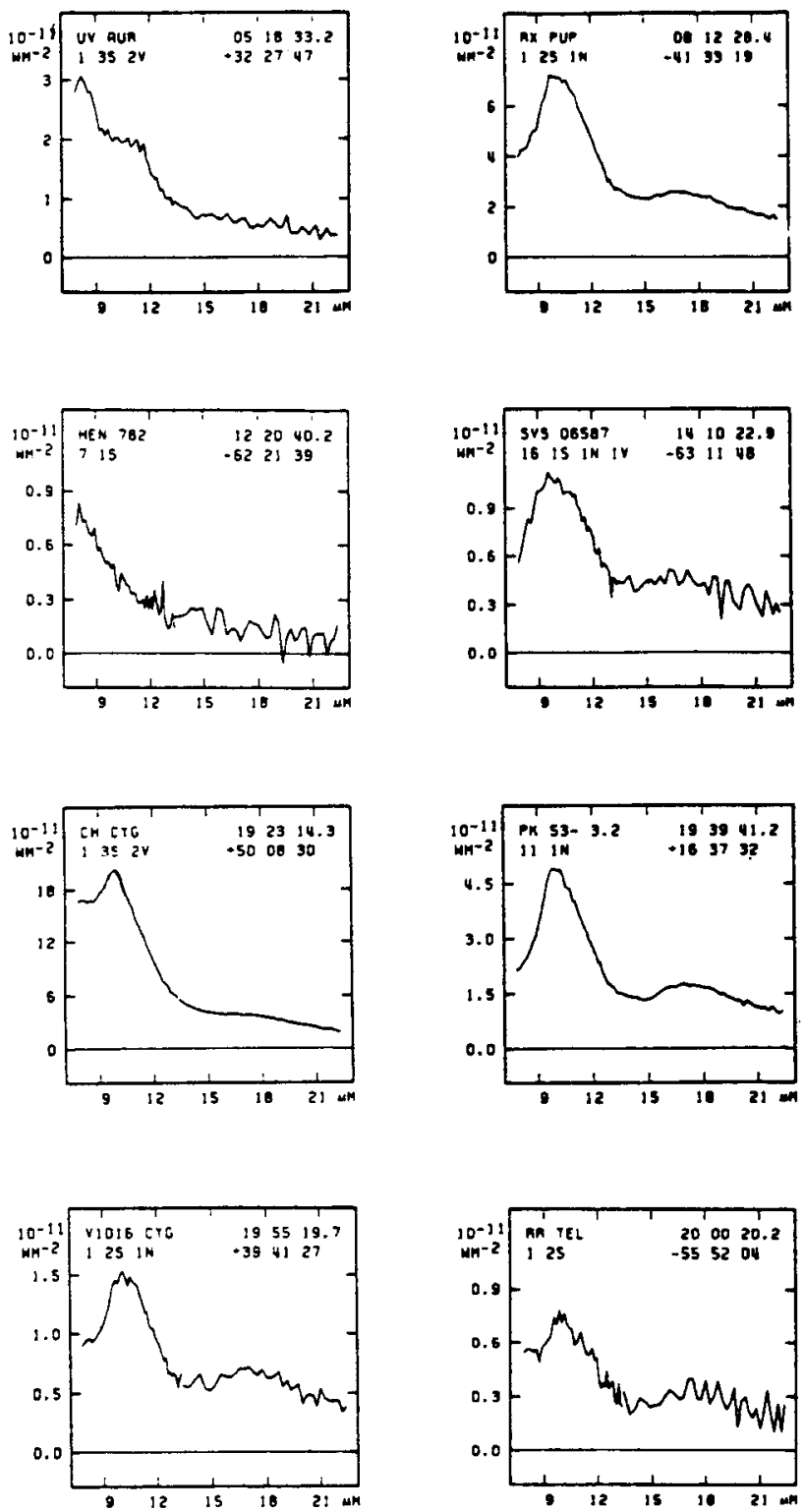

Figure 1. The low resolution 8 to $21 \mu \mathrm{m}$ spectra of symbiotic stars. 


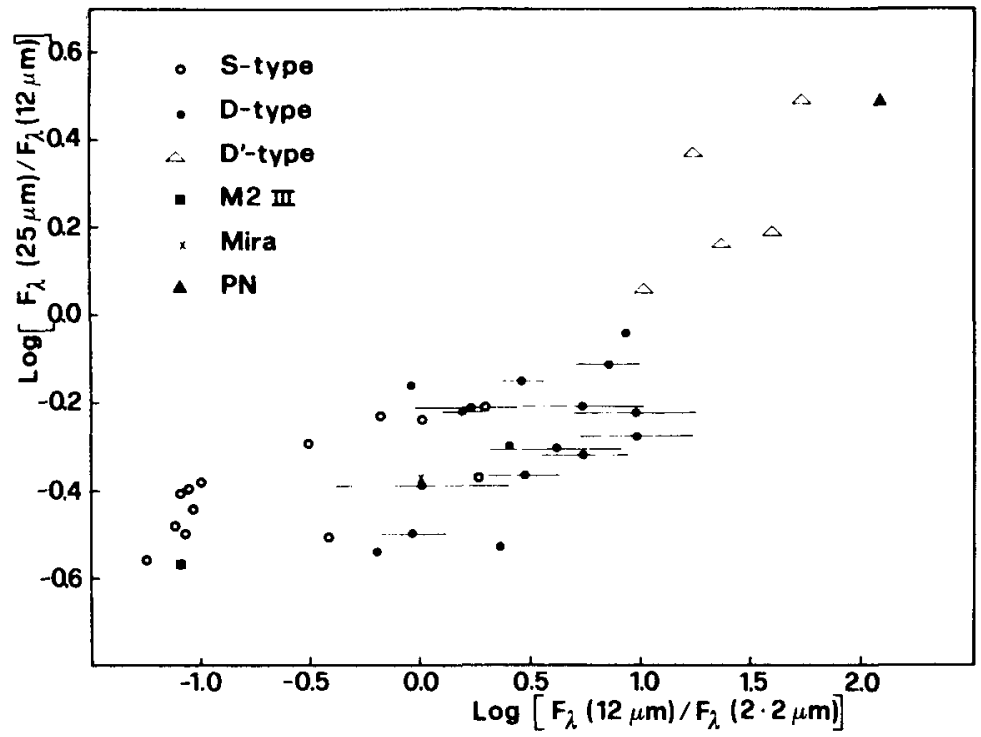

Figure 2. The colour-colour diagram for symbiotic stars. The solid lines indicate the amplitude of light variations of symbiotic Miras.

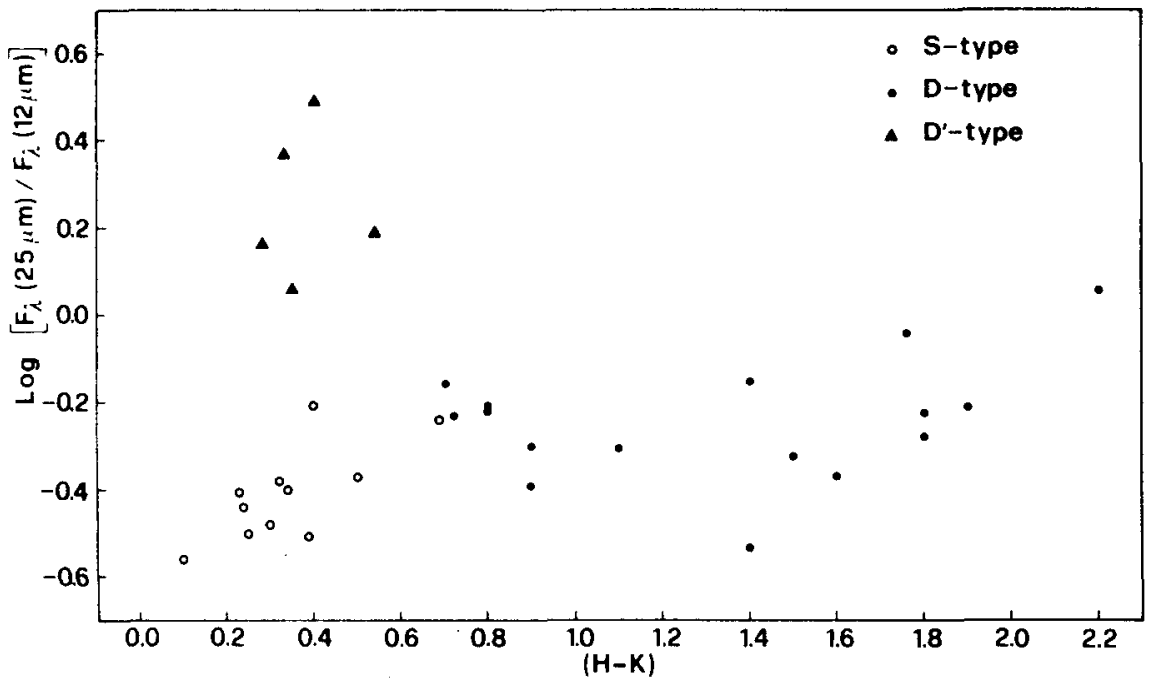

Figure 3. The colour-colour diagram for S, D and D'type symbiotics. 
similar to that observed in normal $M$ giants. However, a few $S$-type symbiotic stars like $\mathrm{CH}$ Cyg have dust envelopes and show relatively higher mass-loss rates. Kenyon, Fernandez-Castro and Stencel (1986) analyzed the IRAS pointed observations of few a S-type symbiotics and found similar results. The LRS spectra of the two S-type symbiotics (Figure 1) $\mathrm{CH}$ Cyg and UV Aur show evidence for 10- $\mu \mathrm{m}$ silicate emission feature. The IRAS fluxes of these two stars also indicate for the presence of dust shells. From an analysis of the data we find that the dust masses and dust temperatures to be of the order of $10^{-6}$ Mo and $10^{-7}$ Me and $500 \mathrm{~K}$ and $500 \mathrm{~K}$ for $\mathrm{CH}$ Cyg and UV Aur respectively. In order to derive the characteristics of the dust shells around S-type symbiotics we need good estimates of distances and effective temperatures of the cool components.

\subsection{SYMBIOTIC MIRAS}

The IRAS data of all symbiotic Miras (D-type) (Table 1) show evidence for the presence of dust envelopes. Pulsation periods are known only for 10 symbiotic Miras (Whitelock, 1987). The period-luminosity relation found from the Mira variables is also found to be applicable to symbiotic Miras (Feast, 1984; Whitelock, 1987). The pulsation periods, $\mathrm{K}$ magnitudes and distances for 10 symbiotic Miras given in Table 2 are from Whitelock (1987). The $M(b o l)$ values are obtained from the relation $\mathrm{M}(\mathrm{bol})=1.06-2.242 \log (\mathrm{P})$ (Feast, 1984). The $L$ values (pulsation amplitude in the $L$ band) are estimated from the light curves and are given in Table 2 . The far-infrared luminosities L(IR) given in Table 2 are derived from the IRAS fluxes (Table 1) and distances (Table 2). The dust temperatures given in Table 2 are derived from the IRAS fluxes. The average dust temperature in the dust envelopes of symbiotic Miras is found to $320 \mathrm{~K}$. The masses of the dust envelopes $M(d)$ are estimated from the equation (Hilderbrand, 1983)

$$
M(d)=4 / 3\left(\operatorname{a\rho d}^{2} f(v) / Q(v) B(v, T(d)),\right.
$$

where $d$ is the distance to the symbiotic Mira, $f(v)$ is the observed flux density (IRAS), $B(v, T(d))$ is the Planck function with dust temperature $T(d), a$ is the radius of the dust particle, and $p$ is the dust grain density. The quantity $a \rho / Q(v)$ is dependent on the dust properties and a value of $\mathrm{a} / \mathrm{Q}(\lambda)(25 \mu \mathrm{m})=1.510^{-3}$ is adopted (Whitelock et al. 1987). The temperatures and masses of the dust envelopes of these ten symbiotic Miras (Table 2) are similar to that found for Mira variables by Whitelock et al. (1987). The dust envelopes areound BI Cru, He2-104 and H1-36 may have temperature gradients. We have not taken the temperature gradients into account. Whitelock et al. (1987) found evidence for pulsationally driven mass loss from Mira.variables. They derived a relation between dust shell mass, pulsation period and pulsation amplitude (at $\mathrm{L}$ band).

$$
\log M(d)(M o)=2.17 \log (P)+1.88 \Delta L-13.38
$$

We obtained the $\Delta L$ values for symbiotic Miras from their light curves (Whitelock, 1987), and from the pulsation periods we estimated the masses of the dust envelopes from the above equation. These values are also listed in Table 2 for comparison. From both these estimates of dust masses we conclude that the masses of the dust envelopes of symbiotic Miras are of the order of $10^{-6}$ to $10^{-7}$ Mo. These values are 
Table 2 :SYMBIOTIC MIRAS

Luminosities Temperatures and Masses of the Dust Envelopes

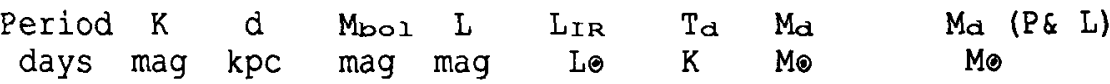

$\begin{array}{lrrlllllll}\text { RX Pup } & 580 & 2.4 & 1.5 & -5.1 & 0.88 & 1761 & 350 & 1.2 \times 10^{-6} & 1.9 \times 10^{-6} \\ \text { He2-38 } & 433 & 4.4 & 3.0 & -4.9 & 0.67 & 330 & 400 & 1.3 \times 10^{-7} & 4.0 \times 10^{-7} \\ \text { BI Cru } & 280 & 4.8 & 2.3 & -4.4 & 0.1 & 547 & 300 & 6.9 \times 10^{-7} & 1.3 \times 10^{-8} \\ \text { He2-104 } & 400 & 6.7 & 4.7 & -4.8 & & 1277 & 220 & 5.6 \times 10^{-6} & \\ \text { He2-106 } & 450 & 4.7 & 2.8 & -4.9 & 0.73 & 1164 & 250 & 3.0 \times 10^{-6} & 5.6 \times 10^{-7} \\ \text { H1-36 } & 500 & 7.4 & 7.6 & -5.0 & & 6476 & 200 & 4.2 \times 10^{-5} & \\ \text { HM Sge } & 540 & 4.2 & 2.3 & -5.1 & 0.7 & 2501 & 375 & 1.3 \times 10^{-6} & 7.3 \times 10^{-7} \\ \text { V1016Cyg } & 450 & 5.1 & 3.4 & -4.9 & 0.5 & 2185 & 300 & 2.8 \times 10^{-6} & 2.1 \times 10^{-7} \\ \text { RR Te1 } & 387 & 4.1 & 2.5 & -4.7 & 0.55 & 565 & 300 & 7.2 \times 10^{-7} & 1.9 \times 10^{-7} \\ \text { R Aqr } & 387 & -1.0 & 0.25 & -4.7 & 0.8 & 241 & 500 & 4.0 \times 10^{-8} & 5.5 \times 10^{-7}\end{array}$

Table 3:YELLOW SYMBIOTIC STARS

Luminosities, Temperatures and Masses of the Dust Envelopes

Sp $\quad K(m a g) \quad d(k p c) \quad L_{I R}(L \theta) \quad T_{d}(K) \quad M_{d}(M \ominus)$

$\begin{array}{llrlrrr}\text { M1-2 } & \text { G2Ib } & 9.8 & 2.0 & 61 & 220 & 2.6 \times 10^{-7} \\ \text { Wray157 } & \text { G } & 9.4 & 2.5 & 51 & 260 & 1.1 \times 10^{-7} \\ \text { AS201 } & \text { G2III } & 9.9 & 2.2 & 77 & 165 & 1.1 \times 10^{-6} \\ \text { Cn1-1 } & \text { F5III } & 7.6 & 0.45 & 40 & 235 & 1.3 \times 10^{-7} \\ \text { HD149427 } & \text { A-F } & 10.3 & 2 . & 150 & 250 & 4.0 \times 10^{-7}\end{array}$


similar to those found for field Mira variables by Whitelock et al. (1987). These results suggest that the mass-loss rate from symbiotic Miras is of the same order of magnitude as that found from Mira variables.

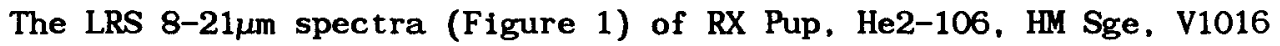
Cyg and RR Tel show the 10pm silicate emission feature. However, the LRS spectrum of BI Cru (Figure 1) shows no 10um silicate emission feature. The 8-21 $\mathrm{m}$ spectrum of $\mathrm{BI}$ Cru is in agreement with the hot grey continuum from dust without any additional silicate component. Roche et al. (1983) made a spectroscopic survey of 22 symbiotic stars in the wavelength region $2 \mu \mathrm{m}$ to $13 \mu \mathrm{m}$. They found that most of the D-types (symbiotic Miras) show the 10um silicate emission feature. Roche et al. (1983) also found a featureless smooth dust emission component. They found no silicate emission feature in BI Cru, AS210, He2-104, and He2-390. IRAS observations (Figure 1, Table 1) of these four symbiotic also shows that they do not have 10 $\mathrm{mm}$ silicate emission feature. The $25 \mu \mathrm{m}$ and $60 \mu \mathrm{m}$ fluxes of $\mathrm{He2}-104$ and $\mathrm{He2}-390$ suggests temperature gradients in their dust envelopes. Roche et al. suggested iron based dust grains to explain the hot grey component in the 2 to $13 \mu \mathrm{m}$ spectra of symbiotic Miras. Whitelock (1987) suggests the presence of large dust grains in the dust envelopes of some symbiotic Miras, which may explain the featureless component in the LRS spectra. But very large particles are not required. According to Draine and Lee (1984) minimum sizes of between $3 \times 10^{-5}$ and $10^{-4} \mathrm{~cm}$ will suffice and can produce neutral extinction.

\subsection{YELLOW SYMBIOTIC STARS}

M1-2, Wray 157, AS201, Cn1-1 and HD149427 show combination spectra (nebular emission lines + underlying stellar absorption spectrum) like the symbiotic stars. But the primary components of yellow symbiotics (D'-type) are of spectral type F-G. The dust temperature is also lower than that observed in symbiotic Miras. All these five objects show near-infrared excess and are also classified as planetary nebulae. In the flux-ratio diagrams (Figures 2 and 3 ) these five objects are well separated from symbiotics and symbiotic Miras. The far-infrared colours and flux distributions (Table 1) of these five yellow symbiotics are similar to those observed in planetary nebulae. In Figure $2 \mathrm{He2-104}$ and H1-36 are in the border region of yellow symbiotics. However, in Figure 3 they are clearly in the region of symbiotic Miras, and yellow symbiotics are well separated from the rest. Cn1-1 shows a F5III spectrum in the optical region with nebular emission lines superposed on it, and the UV (IUE) spectrum suggests the presence of a hot (O-B) companion (Lutz, 1984; Bhatt and Mallik, 1986). Cn1-1 shows strong emission lines characteristic of a high-density planetary nebula (Glass and Webster, 1973). The temperature of the hot (O-B) component is of the order of $70,000 \mathrm{~K}$ (Lutz, 1984; Bhatt and Mallik, 1986). The optical spectrum of AS201 shows characteristics of a G2III star (Kohoutek, 1977, 1987). Kohoutek (1977) considered AS201 as a possible photoplanetary nebula. Feibelman (1988) obtained (IUE) ultraviolet spectrum of AS201 and found evidence for a hot companion in strong CIII, CIV and HeII emissions and a fairly strong UV continuum. He argued that AS2O1 is a planetary nebula and estimated the temperature of the hot companion to be of the order of $60,000 \mathrm{~K}$. Feibelman (1983) also studied the IUE ultraviolet spectrum of M1-2. In the optical region M1-2 shows the 
spectrum of a G2Ib star (O'De1l, 1966). Feibelman (1983) concludes that M1-2 is a young compact high-density planetary nebula of excitation class 5 or 6 , and its nucleus is a binary consisting of a G2Ib star with a hot companion, probably a type 0 subdwarf. Recently Gutierrez-Moreno et al. (1987) studied the optical spectrum of HD149427 and concluded that it is a planetary nebula and not a symbiotic star.

The far infrared (IRAS) colours and flux distributions suggests that these five objects (Table 3) are most likely young compact high-density planetary nebulae. From IRAS data of these five objects we estimated the temperatures, luminosities and masses of the dust envelopes around them (Table 3 ). The masses of the dust envelopes are estimated from equation 1. Based on all the available observations we conclude that M1-2, Wray 157, AS201, Cn1-1, and HD149427 are young planetary nebulae containing a binary nucleus. The evolutionary stage of the late-type $(F-G)$ components is not clear; they may be evolving leftward from the tip of the AGB in the H-R diagram, and the hot components may be subdwarfs or white dwarfs. Another possibility is that the late type (F-G) components are normal giants/supergiants and the dust envelopes are formed from the severe mass loss from the evolved companions which are now hot subdwarfs or white dwarfs. A CNO abundance study of the late-type (F-G) components of these five systems (Table 3) may enable us to understand their evolutionary stage. Kohoutek (1987) finds that the hot components of AS201, M1-2, and Cn1-1 are located on the Harman-Seaton sequence in the region of evolved central stars of planetary nebulae. Kohoutek estimates zanstra temperatures of $78000 \mathrm{~K}$, $72000 \mathrm{~K}$, and $85000 \mathrm{~K}$ for the hot components of AS201, Cn1-1 and M1-2 respectively.

\section{CONCLUSIONS}

Of the 129 symbiotic stars in the Allen's (1984) catalogue 42 were found to be IRAS sources. Only $13 \mathrm{D}$-type and $5 \mathrm{D}^{\prime}$-type symbiotics show flux at $60 \mathrm{~mm}$. The separation of $S, D$ and $D^{\prime}-t y p e s$ into three different groups is clearer in the $\log [\mathrm{f} \lambda(25 \mu \mathrm{m}) / \mathrm{f} \lambda(12 \mu \mathrm{m})]$ versus $(\mathrm{H}-\mathrm{K})$ plot. The $f \lambda(25 \mu \mathrm{m}) / \mathrm{f} \lambda(12 \mu \mathrm{m}) \mathrm{flux}$ ratios and $\mathrm{H}-\mathrm{K}$ colours of SS122, He2-147, AS245, and AS210 suggests that they may be symbiotic Miras. AS210 may contain a carbon star or a Mira. The IRAS fluxes and colours of $S$-type symbiotics stars are consistent with those observed from normal $M$ giants. Excepting CH Cyg and UV Aur, most of the S-type symbiotic stars do not have dust envelopes. These results suggest that mass loss rate from the $M$ giants in S-type symbiotics is similar to that from field normal M giants.

The IRAS data of D-type symbiotic stars (symbiotic Miras) show evidence for the presence of dust envelopes. The dust temperatures and masses of the dust envelopes are estimated for 10 symbiotic Miras with known pulsation periods. The dust temperatures are found to be in the range of 200 to $500 \mathrm{~K}$. The masses of the dust envelopes are of the order of $10^{-6}$ to $10^{-7}$ Me. The dust envelopes in few symbiotic Miras may have temperature gradients.

Analysis of the IRAS data of yellow symmbiotic stars (D'-type) M1-2. Wray 147, AS201, Cn1-1 and HD149427 suggests that they are young high density planetary nebulae containing a binary nucleus. They show evidence for the presence of a hot evolved companion. The evolutionary 
stage of the late type $(F-G)$ companions in these systems is not clear; they may be evolving from the tip of AGB towards left in the $H-R$ diagram.

\section{REFERENCES}

Allen, D.A. 1982, In The Nature of Symbiotic Stars IAU Colloquium No. 70, ed. M. Friedjung and R. Viotti (Dordrecht: Reidel), p. 27.

Allen, D.A. 1984, Proc. Astron. Soc. Australia, 5, 369.

Beichman, C.A., Neugebauer, G., Habing, H.J., Clegg, P.E., and Chester, T.J. 1985, IRAS Point Source Catalogue, JPL.

Bhatt, H.C., Mallik, D.C.V. 1986, Astron. Astrophys. 168, 248.

Draine, B.T. Lee, H.M. 1984, Astrophys. J. 285, 89.

Feast, M.W. 1984, Mon. Not. R. Astr. Soc. 211, 51p.

Feibelman, W.A. 1983, Astrophys. L. 275, 628.

Feibelman, W.A. 1988, in A Decade of UV Astronomy with IUE, ESA SP-281, p. 179 .

Glass, I.S., Webster, B.L. 1973, Mon. Not. R. Astr. Soc. 165, 77.

Gutierrez-Moreno, A. et al. 1987, Preprint Dept. Astro. Univ. Chile.

Hi lderbrand, R.H. 1983, Quart. J. Roy. Astron. Soc. 24, 267.

Kenyon, S.J., Fernandez-Castro, T., Stencel, R.F. 1986, Astron. J. 92, 1118.

Kenyon, S. J., Gallagher, J.S. 1983, Astron. J. 88, 666.

Kenyon, S. J., Webbink, R.F. 1984, Astrophys. J. 279, 252.

Kohoutek, L. 1977, in Planetary Nebulae (IAU Symp. 76) ed. Y. Terzian (Dordrecht: Reide1), p. 47.

Kohoutek, L. 1987, Astrophys. and Space Science 131, 781.

Lutz, J.H. 1984, Astrophys. J. 279, 714.

O'Dell, C.R. 1966, Astrophys. J. 145, 487.

Olnon, F.M., Raimond, E. 1986, Astron. Astrophys. Suppl. 65, 607.

Roche, P.F., Allen, D.A., Aitken, D.K. 1983, Mon. Not ․ Astron. Soc. 204, 1009.

Webster, B.L., Allen, D.A. 1975, Mon. Not. R. Astro. Soc. 171, 171.

Whi telock, P.A. 1987, Publ. Astro. Soc. Pac. 99, 573.

Whitelock, P.A., Pottasch, S.R., Feast, M.W. 1987, in Late Stages of Stellar Evolution, ed. S. Kwok and S.R. Pottasch (Dordrecht: Reidel), p. 269. 